\title{
COMMENT \\ Preventing disease in the twenty-first century: a European Union perspective
}

\author{
Hugo Lagercrantz ${ }^{1}$ and Ariadne Malamitsi-Puchner ${ }^{2}$ \\ Pediatric Research (2020) 87:188-189; https://doi.org/10.1038/s41390-019-0653-8
}

Child health has improved considerably in most of the countries in the European Union during the last decades. The mortality due to prematurity, infectious diseases, cardiac malformations and cancer has never been so low. The infant mortality is lower in most of the European countries than in the USA. However, there are a number of increasing problems like diseases due to lifestyle, misuse of antibiotics, resistance against vaccination. Economic crises like that in Greece affect children in particular. The health care for refugee children is not as good as for the inborn citizens. Neuropsychiatric problems seem to increase. The aim of this article is to encourage pediatricians in the EU to lobby for good health in the EU. Children are usually not organized in unions and they have no votes; therefore, their political influence is weak.

The state of health and the risk for disease in adulthood, largely depend on adverse events taking place during the preconceptional, prenatal, neonatal period and/or first years of life. ${ }^{1}$ The long-term consequences of several stressors happen in the Developmental Origins of Health and Disease (DOHaD) concept, which implies epigenetic changes. The latter are heritable changes in gene expression not affecting the underlying DNA sequence, occurring during phases of developmental plasticity. Thus, the origins of chronic noncommunicable diseases later in life are set before birth as subtle functional changes in gene expression and protein production, cell numbers and metabolism. However, a second, or even a third "hit" or challenge early postnatally and later in life, respectively, might be needed in order to manifest elusive functional changes induced prenatally.

In 2008, the worldwide expanded economic crisis particularly affected Greece. The National Health System, principal provider of health care, was adversely impacted. Consequently, mortality increased, and children's health, who constitute a most vulnerable population group, was seriously affected. ${ }^{2}$

Perinatal studies, comparing precrisis period (before 2008) with periods of early and established crisis (2008-2010/11 and 2011/12 -2014 , respectively), documented decrease in total number of deliveries, higher stillbirth rate, rise in preterm and low birth weight newborns, increased births to women $\geq 35$ years, elevated deliveries by cesarean section and escalation of infant mortality from $2.7 \%$ o to $3.8 \%$ o. However, during crisis, vaccination coverage of indigenous and immigrant population, funded by the state, remained satisfactory. The National Immunization Program was accurately followed, and most vaccines have been timely administered to children. With respect to children's mental health, data during crisis showed alarming increase concerning psychosocial problems and family conflicts. Budget cuts for publicly funded children/adolescent mental health units and community centers resulted in inadequate treatment of children with serious mental disorders. The Greek paradigm clearly indicates that state saving programs should exempt children's/adolescents' somatic and mental care.

Antibiotics are the most commonly prescribed drugs. They are also extensively used to animals in the food industry. Their broad use and often misuse led, besides antibiotic-related adverse events, to multidrug-resistant bacteria (antibiotic resistance), constituting a serious public health issue with global dimensions. ${ }^{3}$

Children suffering from viral infections or from noninfectious diseases are often treated with antibiotics. Further, in cases where narrow-spectrum drugs are indicated, broad-spectrum antibiotics are administered, occasionally with inaccurate total daily dosage, fractioning and longer than indicated treatment duration. To antibiotic misuse count over-prescription of third-generation cephalosporins, carbapenems and quinolones.

Use and misuse of antibiotics differs among Northern and Southern European countries, being much higher in the latter, concerning rate of prescription, overall consumption and broadspectrum penicillin, quinolones and cephalosporins administration. Whether pediatricians or parents pressing for antibiotic prescription are responsible for misuse has been investigated through "knowledge-attitude-practice" questionnaires. Parents of male gender, low education, immigrant or single status, low income, having $<2$ or $>3$ children contribute to inappropriate antibiotic prescriptions. ${ }^{4}$ Nevertheless, reports from Greece and Cyprus, countries with high childhood antibiotic consumption, documented that parents adhere to pediatricians' advice and very seldom acquire antibiotics over the counter. Thus, parents contribute less than expected to antibiotic misuse and relevant reduction could be achieved by educating physicians. Urgent measures to be taken comprise reduction of broad-spectrum antibiotics prescriptions and antibiotic stewardship programs in children's hospitals.

The recent vaccination hesitancy movement spread worldwide. In Europe, divergent political ideologies and antiestablishment parties support movement followers in the name of personal freedom. Such attitudes exerted deleterious consequences with continuous measles outbreaks in several European countries. Regional governments, e.g. Italian Veneto region, suspended mandatory vaccines, aiming at a proactive approach to voluntary vaccine uptake, following health education campaigns. ${ }^{5}$ These policies proved ineffective with a drop of $5.0 \%$ for polio vaccination. The alarming data, forced European countries, as France and Italy to issue laws, making vaccines, as polio and MMR, mandatory for children's admission to nurseries and primary schools. Consequently, vaccination against polio and measles increased, with the

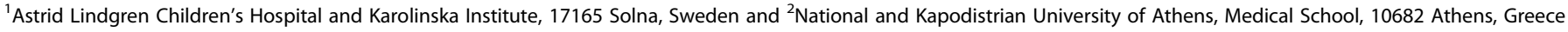
Correspondence: Hugo Lagercrantz (hugo.lagercrantz@ki.se)

Received: 25 September 2019 Revised: 19 October 2019 Accepted: 23 October 2019

Published online: 5 November 2019 
former exceeding in Italian regions 95\% coverage. Important is also timely administration of first and booster shots. Relevant state funding policy ensures best possible results.

Measles outbreaks were recorded among refugees/immigrants in several European countries. In April 2018, 80\% of children in Greek immigrant/refugee camps received the first MMR dose, but coverage dropped to $45 \%$ for the second. All 27 EU countries recommend MMR and polio children vaccination in multiple settings and free of charge.

However, infectious diseases are not any longer the main cause of bad health among children in most of the European countries as it used to be. The main challenge for pediatricians nowadays is obesity, diabetes, environmental allergy and neuropsychiatric conditions. Childhood obesity has increased in most European countries. Metabolic risk factors were found to be higher among Norwegian than in Italian obese children ${ }^{6}$ possibly due to lack of Mediterranean diet in the former. The over consumption of sweet drinks and sweets may be an important factor. Therefore, Norway and Denmark increased the tax for sugar, but not Sweden.

One important cause of childhood obesity is the limited physical exercise and too much screen viewing. ${ }^{7}$ Children do not play and walk to the same extent as they used to.

Children are of course involved in various sporting activities, but only the best players are really supported and those who are not so good are neglected.

Many children spend more than $50 \%$ of their awake time using their tablets, mobiles, playing computer games etc. This does not only restrict the time for physical exercise, but also disturbs sleeping, ability to focus on learning and social interaction.

Although the physical health has improved considerably the psychological health has deteriorated over the last few years in Sweden and probably also in many other EU countries. ${ }^{8}$ Conditions such as attention deficit and hyperactivity disorders (ADHD) and autistic spectrum disorders have increased, although this could be due to increased diagnosis. Depression and anorexia have also increased particularly among girls. ${ }^{9}$ Many youngsters seem to lack reasons to live and injure themselves.

Maybe engagement for the climate change can be good. The successful Swedish climate activist Greta Thunberg (16 years) has admitted that she suffered from anorexia and Asperger syndrome, but now seems to have recovered.

What can pediatricians do to promote child health?

1. Implement the UN Convention of the rights of the child.

2. Defend the best interests of the child.

3. Act as advocates for children, who usually lack strong lobbying units.
4. Try to increase the democratic rights for children. Parents of children below 18 years should get extra votes.

5. Promote physical exercise. Act against closure of playgrounds and sport arenas for children.

6. Limit screen time both at home and in school. The American Academy of Pediatrics has been much more active than corresponding European societies.

7. Introduce sugar tax in all European countries.

8. Obligatory vaccination should be required in the entire EU.

9. The misuse of antibiotics not only to people but also to animals must stop.

10. Promote education from infanthood to adolescence. See article by Johnston in the same issue.

\section{ADDITIONAL INFORMATION}

Competing interests: The authors declare no competing interests.

Publisher's note: Springer Nature remains neutral with regard to jurisdictional claims in published maps and institutional affiliations.

\section{REFERENCES}

1. Hanson, M. \& Gluckman, P. Commentary: Developing the future: life course epidemiology, DOHaD and evolutionary medicine. Int. J. Epidemiol. 45, 993-96 (2016).

2. Malamitsi-Puchner, A. \& Briana, D. D. Economic stress and child health-the Greece experience. Acta Paediatrica published Online first: Epub Date, https://doi.org/ 10.1111/apa.14917 (2019).

3. Principi, N. \& Esposito, S. Antimicrobial stewardship in paediatrics. BMC Infect. Dis. 16, 424 (2016).

4. Bert, F., Gualano, M. R. \& Gili, R. et al. Knowledge and attitudes towards the use of antibiotics in the paediatric age group: a multicenter survey in Italy. Eur. J. Public Health 27, 506-12 (2017).

5. Signorelli, C. \& Odone, A. Four Italian experiences on vaccination policies: results and lessons. Ann. di igiene: Med. preventiva e di comunita 31 (2 Supple 1), 36-44 (2019).

6. Benestad, B., Juliusson, P. B. \& Siegfried, W. et al. Cardiometabolic risk factors differ among adolescents with obesity in three European countries-a cross-sectional study. Acta Paediatrica 108, 493-501 (2019).

7. Radesky, J. S. \& Christakis, D. A. Increased screen time: implications for early childhood development and behavior. Pediatr. Clin. North Am. 63, 827-39 (2016).

8. Lagercrantz, H. Child health in Sweden is characterised by good health and low accidents, but rising psychological problems. Acta Paediatrica 106, 860-63 (2017).

9. Bremberg, S. Mental health problems are rising more in Swedish adolescents than in other Nordic countries and the Netherlands. Acta Paediatrica 104, 997-1004 (2015).

(1)

